\title{
LYTIC LESIONS OF VERTEBRAE WITHOUT INVOLVEMENT OF INTERVERTEBRAL DISC- AN ATYPICAL PRESENTATION OF SPINAL TUBERCULOSIS
}

\author{
HOMAYRA TAHSEEN HOSSAIN ${ }^{1}$, QUAZI TARIKUL ISLAM ${ }^{2}$, MUNSHI MB MD. SHOAIB ADNAN ${ }^{3}$, \\ MUNTASIR IBNE MOBIN ${ }^{4}$, HAM NAZMUL AHASAN ${ }^{5}$, MD. ABUL KASHEM KHANDAKER ${ }^{6}$
}

\begin{abstract}
:
Tuberculosis is very common in our country. It can involve many organs and have broad spectrum of image presentation. It may resemble malignant diseases, especially in the elderly and thus confuse the judgement of the clinician. We report a case of a 58 year old gentleman presenting with chronic cough, low grade fever, weight loss for 16 months; features of progressive marrow failure for 8 months and severe low back pain without any neurological features for 2 months. The CT scan of spine revealed lytic lesions of vertebrae sparing the intervertebral discs, thus resembling metastatic lesions of bone. We approached this case as malignant disease initially, but found it to be a disseminated tuberculosis infection involving lungs, bone marrow and spine after a series of investigations. Though disseminated TB is not uncommon in our country, the purpose of presenting this case is to give emphasis that, clinicians should consider Pott's disease (spinal tuberculosis) in the differential diagnosis of patients with back pain and destructive vertebral lesions. Proper diagnosis and anti-tuberculous treatment with or without surgery will result in cure of the patient.
\end{abstract}

Key words: Tuberculosis; Pott's disease; progressive marrow failure

Received: 03 December 2015

Accepted: 09 June 2016

\section{Introduction:}

Tuberculosis infection (TB) is an important public health problem.Mycobacterium tuberculosis infection can involve many organs. The lung is the most common lesion site but extrapulmonary involvement is not uncommon especially in immunocompromised patient.

Tuberculosis remains a major cause of bone and joint infection in developing countries.A delay in diagnosis and timely initiation of treatment of aggressive TB manifestations like vertebral TB may cause severe andirreversible neurologic sequel including paraplegia, even if anti-mycobacterial chemotherapy is available $^{1}$.

TB of the spine is an ancient disease. In 1782, Sir Percival Pott described spinal TB and surgical treatment of paravertebral abscess. Hence, spinal TB was called 'Pott's Disease'. Spinal TB accounts for $50 \%$ of the cases of skeletal TB, $15 \%$ of the cases of extrapulmonary TB and $2 \%$ of all cases of $\mathrm{TB}^{2-4}$. The lower thoracic and thoracolumbar spine are the most common areas involved, comprising $48 \%$ to $67 \%$ of lesions $^{5}$.

Spinal tuberculosis in its classical form (i.e., "twovertebra diseasewith the destruction of the intervening intervertebral disc and aparavertebral or psoas abscess") is easily recognized and readily treated $^{6}$. in areas where tuberculosis is endemic ${ }^{7}$. Atypical spinaltuberculosis without the aforementioned clinical or radiographic features, occur less frequently ${ }^{8}$.

The purpose of this case report is to highlight that, without involving intervertebral disc, spinal tuberculosis can occur and can mimic metastatic bone

1. Assistant Professor, Medicine, Popular Medical College Hospital, Dhaka

2. Professor of Medicine,Popular Medical College Hospital, Dhaka

3. Registrar, Medicine,Popular Medical College Hospital, Dhaka

4. Assistant Registrar, Medicine,Popular Medical College Hospital, Dhaka

5. Professor of Medicine, Popular Medical College Hospital, Dhaka

6. Professor \& Head, Department of Medicine, $\mathrm{PMCH}$

Address of correspondence: Dr. Homayra Tahseen Hossain (FCPS, MRCP), Assistant Professor, Medicine, Popular Medical College Hospital, Dhaka. Email: homayra.tahseen@ gmail.com

Bangladesh J Medicine 2016; $27: 81-85$ 
lesion. We should always try to reach histopathological / microbiological confirmatory diagnosis.

\section{Case report:}

A 58 years old nondiabetic, normotensive, exsmokergentleman, hailing from Dhaka, was reasonably alright about 16 months back ( February'2014). At that time, he developed dry cough with low grade fever for 2-3 weeks. There was evening rise of temperature with night sweats, but temperature was not documented. There was one episode of haemoptysis. He had anorexia \& some amount of weight loss. His bowel \& bladder habit was normal. He was treated with one course of antibiotic (14 days), but he did not improve. He continued to suffer from fever $\&$ weight loss. At that time, his CXR- (Figure-01)

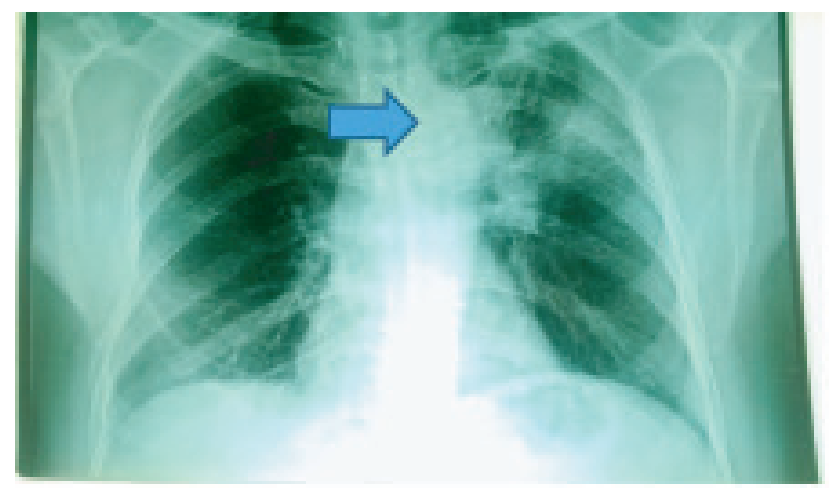

Fig.-1: CXR P/A view (Feb'2014)-

Dense inhomogeneous opacities are noted in left upper \& mid zone. Inhomogeneous opacity also noted right upper zone.

He had a history of tubercular pleural effusion in 1983 \&completed 6 months category-I antiTB
drugs.On the basis of clinical features, past history\& CXR findings, anti-tubercular drug was startedon March'2014. But, he developed severe hypersensitivity reactions just after 2 days of starting anti-TB drugs in the form of generalized extensive urticaria. He stopped taking the drugs.

On April'2014, he received another course of oral antibiotic. This time, his fever \& cough improved. He also regained his appetite. About 4 months later, during routine follow up, his CBC revealed neutropenia with relative lymphocytosis. At that time, he had no chest symptoms, but there was a mild consolidation (left) in his CXR. Gene xpert did not detect MTB from sputum.

He was referred to a haematologist. Bone marrow study revealed reactive hyperplasia. He was started receiving injectable GM-CSF twice/ week. Every time he did $\mathrm{CBC}$, there was leucopenia.

About 5-6 months later, during April'2015, he developed severe agonising low back pain. There was no radiation of pain. There was local tenderness over lower part of the spine. There was no neurological deficit.

Due to severe low back pain, recurrence of fever \& progressive neutropenia, he was hospitalized for total evaluation. He lost $13 \mathrm{~kg}$ weight during last 1 year of illness (highest in last 2 months). During hospital stay, he developed high grade temperature $\left(105^{\circ} \mathrm{F}\right)$. His general conditions progressively deteriorated. Other than back pain, no systemic symptom was present. Repeated clinical examination did not give any clue except anaemia $\&$ high temperature. There was no lymphadenopathy or organomegaly. Neurological examination was normal.

Keeping in mind the possibility of haematological malignancy or skeletal metastasis, thorough investigations were done.

\section{Complete blood count with comment on PBF:}

\begin{tabular}{lccccclll}
\hline Date & Hb\% & TC & \multicolumn{2}{c}{ DC } & Platelet & ESR & PBF & Remarks \\
& & & N\% & L\% & Count & \\
\hline 20.08 .14 & 13.10 & 2600 & 37 & 57 & $1,60,000$ & 58 & leucopenia & $1^{\text {st }}$ detection of leucopenia \\
23.05 .15 & 09.80 & 1400 & 37 & 61 & $1,53,000$ & 88 & Bicytopenia & \\
& & & & & & & With rouleaux & formation \\
12.06 .15 & 09.70 & 930 & 41 & 55 & 64,000 & 74 & pancytopenia & Lowest count of WBC \\
\hline
\end{tabular}

(NB- Hb- Haemoglobin, TC- Total count of WBC, DC- Differential count of WBC, N- Neutrophil, L-Lymphocyte) 
2. Bone marrow examination (27.04.15):> 30\% of marrow nucleated cells are mature and precursor lymphocytes. Features are suggestive of lymphoid disorder.

3. Bone marrow for immunohistochemistry markers (20.05.15): cellular reactive marrow. No evidence of infiltration by atypical lymphoid cells seen.

4. CXR P/A view (Fig-02)

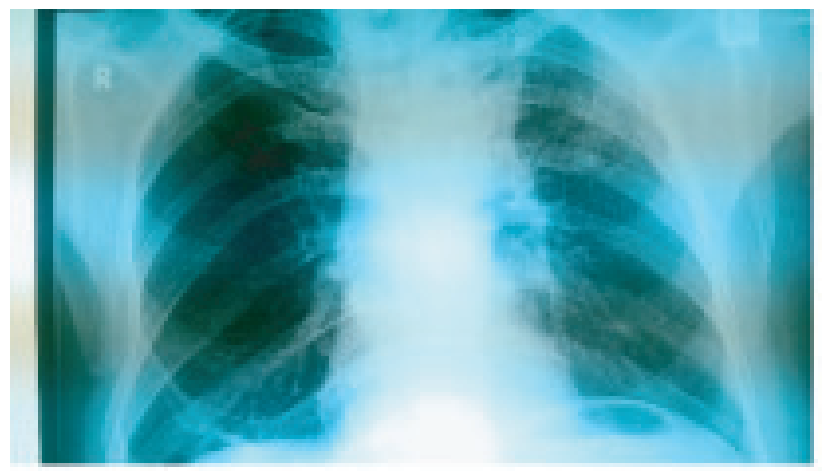

Fig.-02: CXR P/A view (June'2015):

Ill-defined soft irregular opacity is seen in left upper zone \& to some extent in right upper zone.

Impression:? Chronic pulmonary tuberculosis (bilateral)

(Suggest reactivation)

\section{CT scan of chest (Fig-03)}

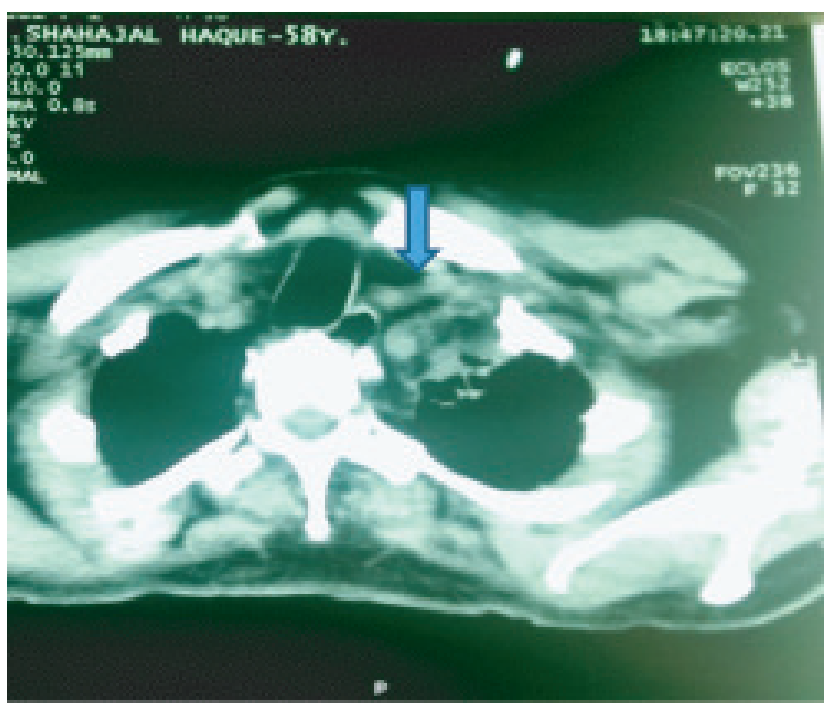

Fig.-03: HRCT Chest (June'2015): CT features are consistent with the sequel of chronic inflammatory process with possible (?) localized fibrosis at left upper lung field.
6. CT Guided FNAC from lung upper left lesionInflammatory lesion

7. RFT/ LFT/ RBS/Cardiac function- Within normal limit

8. ICT for malaria- Negative

9. Tuberculin test $-02 \mathrm{~mm}$

10. HIV 182- Negative

11. Gene xpert for detection of Mycobacterium tuberculosis(specimen- sputum)(June'2015): MTB detected.

12. X-ray lumbosacral spine (Fig-04):

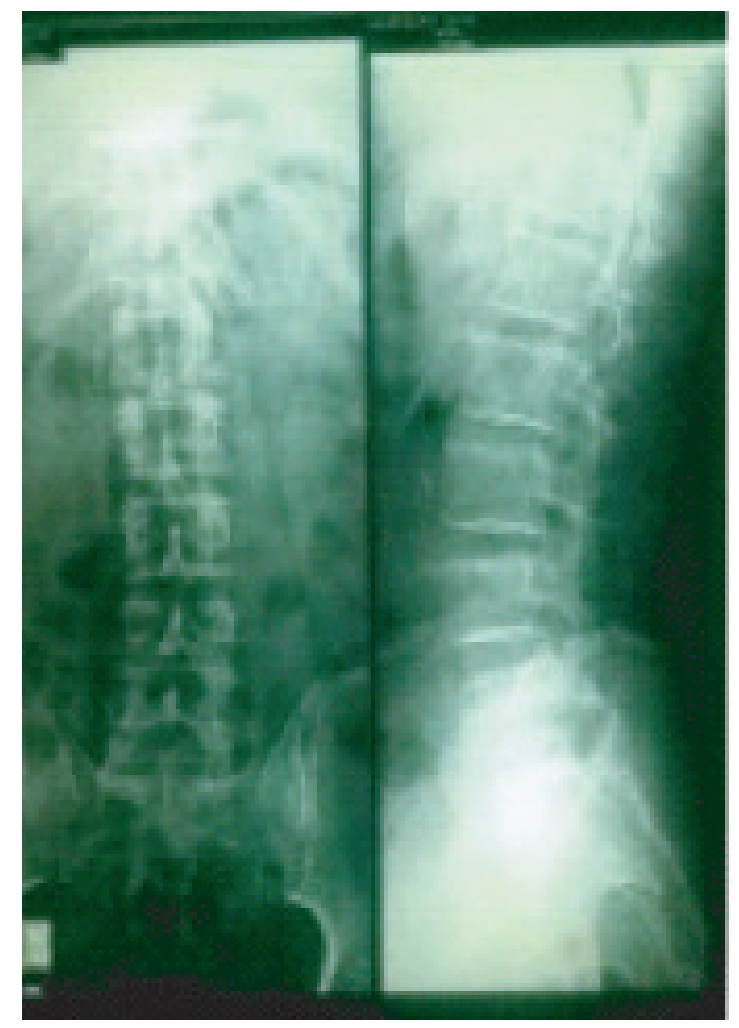

Fig.-04: x-ray of lumbo-sacral spine- AP \& lateral view:

Degenerative change at lumbar spine

13. CT scan of Abdomen (Fig-05)

14. PSA- normal

15. Plasma protein electrophoresis- Polyclonal hypergammaglobulinemia. No underlying monoclonal band is noted.

16. CT guided needle biopsy from vertebral lesion (L4) \& right paravertebral mass lesion 

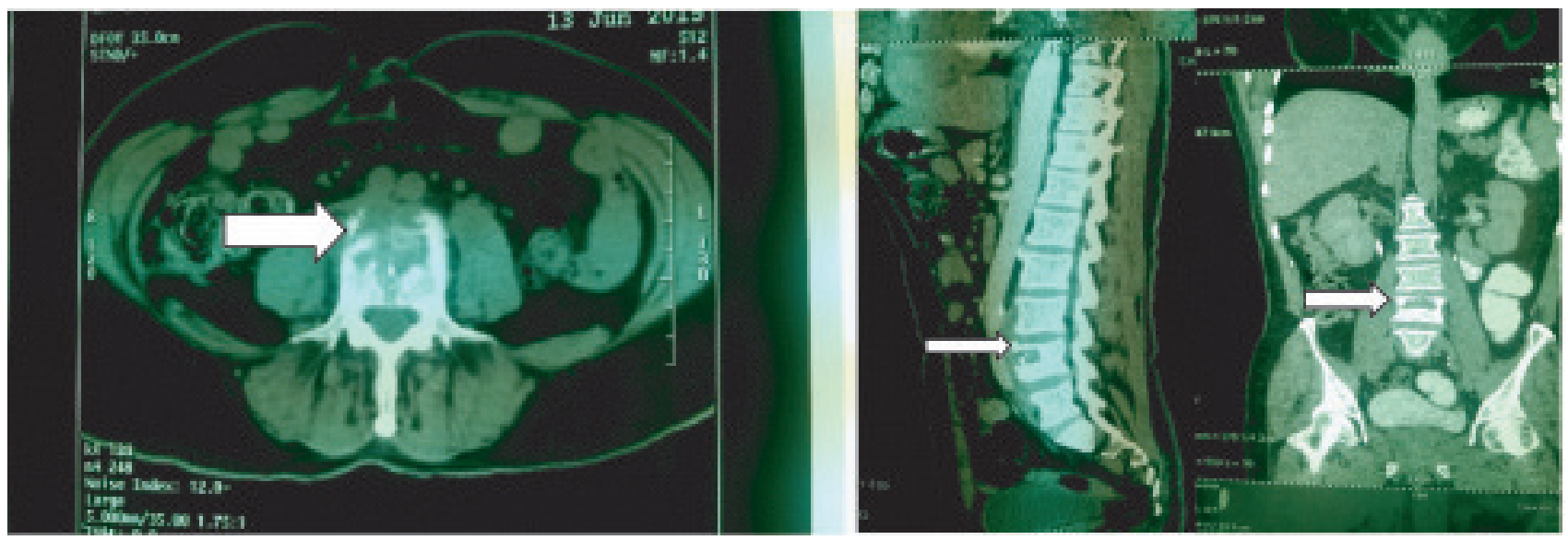

Figure-05:CT scan of Abdomen (June'2015): Lytic lesion in L4 \& L2 vertebral body with mild right paravertebral mass@ @4 level- suggestive of metastatic lesion. Intervertebral disc intact.

(Fig-06) -suggestive of granulomatous inflammation, tubercular.

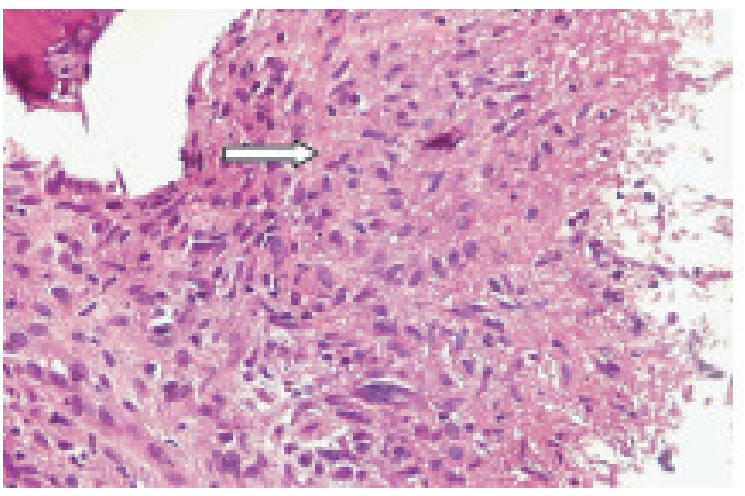

Fig.-06: CT guided needle biopsy from vertebral lesionsuggestive of granulomatous inflammation, tubercular.

17. Bone marrow for AFB culture - sent, but report pending.

After series of investigations, our final diagnosis: Disseminated tuberculosis involving lungs (reactivation of TB), bone marrow (indirectly proven, waiting for bone marrow culture report for microbiological confirmation) \& vertebral body.

Anti-tubercular drugs (3 drugs regime-INH, Rifampicin \&Ethambutol) without Pyrazinamide(due to $\mathrm{H} / \mathrm{O}$ hypersensitivity) was started. The patient's condition started improving gradually. One month after initiating anti-TB therapy, the patient became afebrile, gained $2 \mathrm{~kg}$ body weight \& total count of WBC became $3400 / \mathrm{cmm}$ without Granulocyte Colony Stimulating Factor. All clinical \& cytological parameters improved.

\section{Discussion:}

Tuberculosis is very common in our country including disseminated infection. Still, we report this case to highlight two important points. One is to show how difficult sometimes it could be to differentiate active tubercular lesion from old healed lesion, thus the necessity of starting treatment or not. Second important point is the involvement of vertebrae with lytic lesion sparing intervertebral disc can be a presenting feature of spinal TB, though very uncommon and confusing with metastatic lesion.

In spinal $\mathrm{TB}$, onset of symptoms is usually insidiousand disease progression slow. The usual presentationconsists of pain overlying the affected vertebrae, low-grade fevers, chills, weight loss, and nonspecificconstitutional symptoms of varying duration. Paraplegia can be the first sign of spinal disease. Varyingdegrees of weakness, nerve-root compression and sensory involvement can occur. Duration of symptomsprior to diagnosis ranges from 2 weeks to several years. Weight loss has been recorded in $58 \%$ of patients ${ }^{9}$ and $90 \%$ to $100 \%$ of patients had back pain ${ }^{10}$.

A confident radiological diagnosis is made in lessthan half of the cases, with the principal alternativediagnosis being malignant disease. The relative merits of different imaging procedures like plain radiography, CT and MRI in the diagnosis of spinal TB have beenevaluated. Most of the studies have failed to describehow much information is provided by each test. Conventional radiographs give a good overview; CTvisualizes the disco-vertebral lesions and paravertebral abscesses, whileMRI is useful in determining the spreadof the disease to the soft tissues and spinal canal ${ }^{11}$. Theplain radiograph described changes consistent with TB spine in $91-99 \%$ of cases $^{12}$.

The best diagnostic modality for spinal TB is MRI ${ }^{10,11}$. In our case, CT scan of abdomen was done for 
abdominal organs \& spine together. As we could confirm our diagnosis by CT scan, we did not go for MRI.The radiographic features of typical tuberculous spondylitisinclude narrowing of the intervertebral disk spaces and destructionof the contiguous vertebral bodies.

Excellent results with treatment of TB spine can beachieved if early diagnosis is made. Increasing backpain should suggest plain radiography of the spine, and perhaps followed by MRI.

Differentiating TB of the spine from osteoporotic compression fractures of the spine, and from spinal cordinvolvement in malignancies is important. Timelytreatment of spinal TB can avoid extensive investigations, treatment delays and adverse longterm out-comes, including compression fractures with neurological deficits ${ }^{13}$.

\section{References:}

1. Turgut M: Spinal tuberculosis (Pott's disease): its clinical presentation, surgical management, and outcome. A survey study on 694 patients. Neurosurg Rev 2001, 24:8-13.

2. Fancourt GJ, Ebden P, Garner P. Bone tuberculosis: results and experience in Leicestershire. $\mathrm{Br} \mathrm{J}$ Dis Chest 1986; 80: $265 \pm 272$.

3. Naim-ur-Rahman (1980) Atypical forms of spinal tuberculosis. J Bone Joint Surg Br 62: 162-5.

4. Watts HG, Lifeso RM (1996) Tuberculosis of bones and joints. J Bone Joint Surg Am 78: 288- 298.
5. Yalniz E, Pekindil G, Aktas S (2000) Atypical tuberculosis of the spine. Yonsei Med J 41: 657661

6. Wolfgang GL. Tuberculosis Joint Infection. ClinOrthopRel Res 1978; 136: 257 -263.

7. Davies PD, Humphries MJ, By ${ }^{\circledR e d ~ S P . ~ B o n e ~ a n d ~}$ Joint Tuberculosis. A survey of notifications in England and Wales. J Bone Joint Surg (Br) 1984; 66: $326-330$.

8. Dobson J. Tuberculosis of the spine. An analysis of the results of conservative treatment factors influencing prognosis. J Bone Joint Surg (Br) 1951; 33: $517 \pm 531$

9. Pertuiset E, Johann B, Liote F. Spinal tuberculosis in adults. A study of 103 cases in a developed country, 1980 -1994. Medicine 1999; 78: 309 - 320.

10. Azzam NI, Tammawy M. Tuberculous spondylitis in adults: diagnosis and treatment. $\mathrm{Br} J$ Neurosurg1988; 2: 85- 91.

11. Lindhal S, Nymann RS, Brismar J. Imaging of tuberculosis. IV. Spinal manifestations in 63 patients. ActaRadiol1996; 37: 506 - 511.

12. Jain R, Sawhney S, Berry M. Computed tomography of tuberculosis: patterns of bone destruction. ClinRadiol1993; 47: 196 - 199.

13. B Dass, TA Puet, C Watanakunakorn. Tuberculosis of the spine (Pott's disease) presenting as 'compression fractures'. Spinal cord(2002) 40, 604608 